\title{
Isolation, Identification, and Antimicrobial Profiling of Bacteria from Aquaculture Fishes in Pond Water of Bangladesh
}

\author{
Mohammad Zakerin Abedin ${ }^{1}$, Md. Sadiqur Rahman ${ }^{2}$, Rubait Hasan ${ }^{3}$, Jamiatul Husna Shathi ${ }^{4}$, Laila \\ Jarin $^{5}$, and Md. Sifat Uz Zaman ${ }^{6}$ \\ ${ }^{1}$ Dept. of Microbiology, Khwaja Yunus Ali University, Sirajgonj, Bangladesh; ${ }^{2}$ Dept. of Microbiology, Aqua Laboratory \\ Quality Feeds Limited, Mymensingh, Bangladesh; ${ }^{3 \& 4}$ Dept. of Biochemistry and Biotechnology, Khwaja Yunus Ali \\ University, Sirajgonj, Bangladesh; ${ }^{5}$ Dept. of Microbiology, LabAid Medical Centre Gulshan Ltd, Dhaka, Bangladesh; and \\ ${ }^{6}$ Dept. of Microbiology, Popular Diagnostic Centre Ltd, Dhaka, Bangladesh \\ *Correspondence: zakerin.du2016@gmail.com (Mohammad Zakerin Abedin, Assistant Professor, Dept. of Microbiology, \\ Khwaja Yunus Ali University, Bangladesh)
}

\begin{abstract}
Bacterial diseases are widespread and can be of particular importance in fish farming of Bangladesh. This investigation was done to assess and compare the bacteria diversities and population in local fresh water pond fishes. Out of 95 samples, 54(56.9\%) were Shing (Heteropneustes fossilis), 14(14.7\%) were Pangas (Pangasius pangasius), 9(9.5\%) were Pabda (Ompok spp), 7(7.3\%) were Thai Koi, (Anabas testudineus), and 11(11.6\%) were others infected fishes such as Shol (Channa striata), Magur (Clarias spp), Tilapia (Oreochromis niloticus), and Tengara (Mystus cavasius) fishes. Among 95 infected fishes 84(88.4\%) were infected with pathogenic bacteria and 11(11.6\%) were normal flora. There were eight types of different isolated spp with frequency of occurrence were 36 (42.9\%) Aeromonas spp, 15 (17.9\%) Pseudomonas spp, 7(8.3\%) Vibrio spp, 9 (10.7\%) Staphylococcus spp, 7(8.3\%) Flavobacterium spp, 7(8.3\%) Edwardsiella spp, others were 3(3.6\%) Citobacter spp, and Enterobacter spp respectively. All of the isolated pathogenic bacteria showed 84/84(100\%) resistant to Amoxicillin and 18/84(64.3\%) resistant to Erythromycin. All the strains showed sensitive to Ciprofloxacin, Cotrimoxazole, Enorfloxacin, Doxyciline, Clotetracycline, and Colistin with the frequency of occurrence were 78/84(92.8\%), 76/84 (90.5\%), 73/84(86.9\%), 67/84(67.9\%), 53/84(63.1\%) and 52/84(61.9\%) respectively. The physiochemical characteristics of 95 pond water samples were analyzed; the average water temperature, $\mathrm{p}^{\mathrm{H}}$, and ammonia were $27.3^{\circ} \mathrm{C}, 7.6$ and $0.87 \mathrm{mg} / \mathrm{L}$ respectively. The significant variation in the physiochemical parameters like water temperature, $\mathrm{p}^{\mathrm{H}}$, ammonia were observed within these five types of ponds water. Hence, it is important to detect fish diseases, responsible pathogens and other agents for protection of our water resources.
\end{abstract}

Keywords: Fresh water fishes, Pathogenic microorganisms, antibiotic sensitivity, physiochemical parameters.

\section{INTRODUCTION}

The fresh water fishes are well known and highly valuable fish species in Bangladesh. Among them, Shing (H. fossilis), Pangas (P. pangasius), Thai Koi

UniversePG I www.universepg.com
(A. testudineus), Pabda (Ompok spp), Shol (C. striata), Tengara (M. cavasius), Magur (Clarias spp), and Tilapia (O. niloticus) are very popular in Asian region. A wide range of bacterial pathogens were associated 
with fishes specially, with the aquaculture fishes in ponds. The naturally living in the fish's habitat is reservoir of the indigenous bacterial pathogens such as Aeromonas spp and Vibrio spp. There are some factors that induce bacteria to be pathogens such as poor water quality and physiologically uneven fish which permit opportunistic bacterial diseases to win (Austin, 2011; Rahman et al., 2019).

A number of pathogenic bacteria are related with fish and shellfish for instance Streptococcus spp, Aeromonas spp, Vibrio spp, Flabobacterium spp and others pathogens to cause diseases. The economic losses because of fish mortality are mainly caused by various pathogenic bacteria and other microorganisms in Bangladesh (Md et al., 2014). In the fishes there are two types of bacteria like indigenous bacteria and nonindigenous microbes. Staphylococcus aureus, Escherichia coli, Clostridium botulinum, Listeria monocytogenes are some examples of non-indigenous microorganisms and then again, indigenous microbes incorporate Aeromonas spp., Vibrio spp., Salmonella spp, and Pseudomonas spp. In Bangladesh, generally fresh water fishes are found in little rivers, swamp and canal. Now a day's commercial fish cultivating in lake is exceptionally well known. The dangerous microbes such as Pseudomonas spp, Aeromonas spp, Staphylococcus spp, Flavobacterium spp, Citobacter spp Edwardsiella spp, and Vibrio spp that live in every pond causing perilous bacterial diseases, for example, ulcer, blade decay and tail spoil of fishes (Uddin et al., 2017).

The poor water quality is also a great reason that consumes off the sludge coat or stresses the fishes making them progressively powerless to bacterial infection. Therefore, this study was designed with a view to assess the occurrence of bacterial infection of ponds cultivated fishes and their respective ponds water quality assessment and antibiotic susceptibility analysis against bacterial pathogens of fishes.

\section{MATERIALS AND METHODS}

2.1. Collection and transportation of samples - A total of 95 infected fishes including Shing (H. fossilis), Pangas (P. pangasius), Thai Koi (A. testudineus), pabda (Ompok spp) and others like Shol (Channastriata), Magur (Clarias spp), Tilapia

UniversePG I www.universepg.com
(Oreochromisniloticus), and Tengara (M. cavasius) and with their water samples were taken from different 18 Upazila in greater Mymensingh, Netrokona, and Sherpur districts in Bangladesh between April 2019 to December 2019. During the collection of fish samples and water precautionary measures were maintained to avoid touch and ice box were used to maintain cool chain. The samples were then brought to the laboratory of the Quality Aqua Laboratory, Quality feeds Limited, Mymensingh.

\subsection{Sample processing and enrichment of bacteria -}

Aseptic measures were undertaken during the sampling procedure to prevent contamination of the samples. Three types of specimens such as intestine, skin and gill of infected fishes were collected for microbiological test. These specimens were taken in a sterile chopping board and then minced properly and grinded together. Ten (10) gm of samples were homogenized with 90 milliliters $(\mathrm{ml})$ of freshly prepared $0.1 \%$ peptone water and $0.1 \mathrm{ml}$ of homogenized sample was inoculated according to standard methods on to selective media such as: Rimler Shotts Medium Base agar (for Aeromonas spp.), Pseudomonas Base agar (for Pseudomonas spp), Thiosulfate citrate bile salt sucrose (TCBS) agar (for Vibrio spp.), Tryptic Soy Agar (TSA) for enrichment of bacterial isolates, Brain Heart Infusion (BHI) Agar (for fastidious organisms) and finally incubated at $37^{\circ} \mathrm{C}$ for 24 hours.

2.3. Identification of bacterial pathogens - Suspected bacterial colonies obtained from different culture plates were isolated and then streaked on TSA slants, MIU medium, Simon citrate agar stant and incubated overnight at $37^{\circ} \mathrm{C}$. The pure isolates were characterized by bacterial cell morphology, alkaline and acidic reaction, $\mathrm{H}_{2} \mathrm{~S}$ (hydrogen sulfide production) and gas production, motility test, indole production, urease test, oxidase test, catalase test, Methyl Red (MR) test, and Voges Praskaure (VP) test.

The Gram staining techniques were performed to identify Gram positive and Gram negative bacteria. The biochemical tests were carried out to identify the pathogens following Bergey's manual of Bacteriological classification (John et al., 1998). 
Abedin et al., / American Journal of Pure and Applied Biosciences, 2(3), 39-50, 2020

2.4 In-vitro antimicrobial sensitivity test - According to the CLSI guidelines (CLSI, 2015), the Kirby- Bauer disc diffusion methods were used to in-vitro antimicrobial susceptibility tests of all the pathogenic bacteria isolates. The commonly used antibiotics were: Amoxicillin $(10 \mu \mathrm{g})$, Ciprofloxacin $(5 \mu \mathrm{g})$, Colistin $(25 \mu \mathrm{g})$, Clotetracyclin $(30 \mu \mathrm{g})$, Doxyciline $(30 \mu \mathrm{g})$, Erythromycin $(15 \mu \mathrm{g})$, Cotrimoxazole $(25 \mu \mathrm{g})$, and Enrofloxacin $(5 \mu \mathrm{g})$. Aeromonas hydrophila (ATCC 7966), Pseudomonas aeruginosa (ATCC 27853), Staphylococcus aureus (ATCC 25923), Vibrio cholerae (ATCC 14035), and Flavobacterium columnare (ATCC 23463) were used as quality control throughout the study for culture and antimicrobial susceptibility testing. The suspected isolated bacterial colonies were taken in sterile PBS (phosphate buffered saline) water and then adjusted to 0.5 McFarland's turbidity standard. The bacterial suspension was spread onto Mueller-Hinton agar (Himedia, India) and then impregnated antibiotic discs (Himedia, India) were placed and incubated at $37^{\circ} \mathrm{C}$ for 24 hours. Around the discs, the antibiotic zones of inhibition conformed were estimated in diameter of millimeter $(\mathrm{mm})$. The zone span was really scaled from the focal point of the anti-microbial plate as far as possible of the reasonable zone where microscopic organisms could be seen developing. The interpretation of antibiogram was measured in millimeter $(\mathrm{mm})$ of diameters as sensitive, intermediate and resistant as per the producer's guidelines.

2.5 Water physio-chemical parameters - Ponds water temperature and $\mathrm{pH}$ were analyzed by the digital thermometer and digital $\mathrm{pH}$ meter (Hanna instruments, USA) respectively. The ideal value of temperature and $\mathrm{pH}$ was $30-32^{\circ} \mathrm{C}$ and 7.0 - 8.5 separately. Ammonia, nitrate, and nitrite were determined by colorimetric method by using Rapid Hanna Test kit (Hanna instruments, USA), with a special smallest increment range of $0.5 \mathrm{mg} / \mathrm{L} \mathrm{NH} \mathrm{NH}_{3}-\mathrm{N},<10 \mathrm{mg} / \mathrm{L} \mathrm{NO}_{3}-\mathrm{N}$, and < $0.2 \mathrm{mg} / \mathrm{L} \mathrm{NO}_{2}-\mathrm{N}$ respectively. The total hardness and alkalinity were measured by EDTA, phenolphthalein, and bromophenol titration by using Rapid Hanna Test Kit (USA) with a special ideal value of $20-100 \mathrm{mg} / \mathrm{L}$ and $50-160 \mathrm{mg} / \mathrm{L}$ separately.

2.6 Statistical analysis of experimental data - Data obtained were analyzed by SPSS version 20 and Excel
2016. Descriptive statistics and chi-square tests were done to check the statistical evaluation. The p-value that considered as significant was $<0.5$.

\section{RESULTS:}

\subsection{Clinical Signs and post mortem findings - After} clinical examination of diseased fishes, several severe damages were observed such as equilibrium loss, hemorrhagic ulcerative lesion, rectal protrusion, dropsy, body and tail erosion, reddish discoloration around the eye and mouth, skin lesions on body surface, and profuse mucous secretion (Fig 1). In postmortem examination of diseased fishes, organ enlargement and congestion in internal organs were also appeared.

\subsection{Bacteria isolated from different diseased fishes}

- The isolated bacterial pathogens were founded from different infected fishes are presented in Table 1. Out of 95 infected fishes samples, 84(88.4\%) were infected with pathogenic bacteria and $11(11.6 \%)$ were normal flora. Out of 84 infected fishes spp, 47(49.5\%) were Shing (H. fossilis), 13(13.7\%) were Pangas, 6(6.3\%) were pabda (Ompok spp), 7(7.3\%) were Thai Koi $(A$. testudineus), and other 11(11.6\%) infected fishes were Shol, Gulsha, Magur, Tilapia, and Tengra fishes. Among the total isolation of 84 bacterial strains, the highest number was 36(42.9\%) for Aeromonas spp, the second highest for Pseudomonas spp, was 15(17.9\%), and the next was $9(10.7 \%)$ for Staphylococcus spp. On the other hand, only 7(8.3\%) spp of Vibrio spp., Flavobacterium spp., Edwardsiella spp and the rest of 3(3.7\%) Citobacter spp, and Enterobacter spp.

\subsection{Biochemical tests for bacterial identification -} The identification of the pure bacterial isolates were performed by biochemical parameters included alkaline reaction, acidic reaction, $\mathrm{H}_{2} \mathrm{~S}$ (hydrogen sulfide production) gas production, motility test, indole production, urea hydrolysis, catalase test, oxidase test, Methyl-Red (MR) test, Voges-Praskaure (VP) test are presented in Table 2.

In all of the isolated strains it was observed that only one strain was found as Gram positive Cocci and the rest 7 isolates were found as Gram negative bacteria. The biochemical analysis revealed that all of the strains were catalase negative in our experiment. 


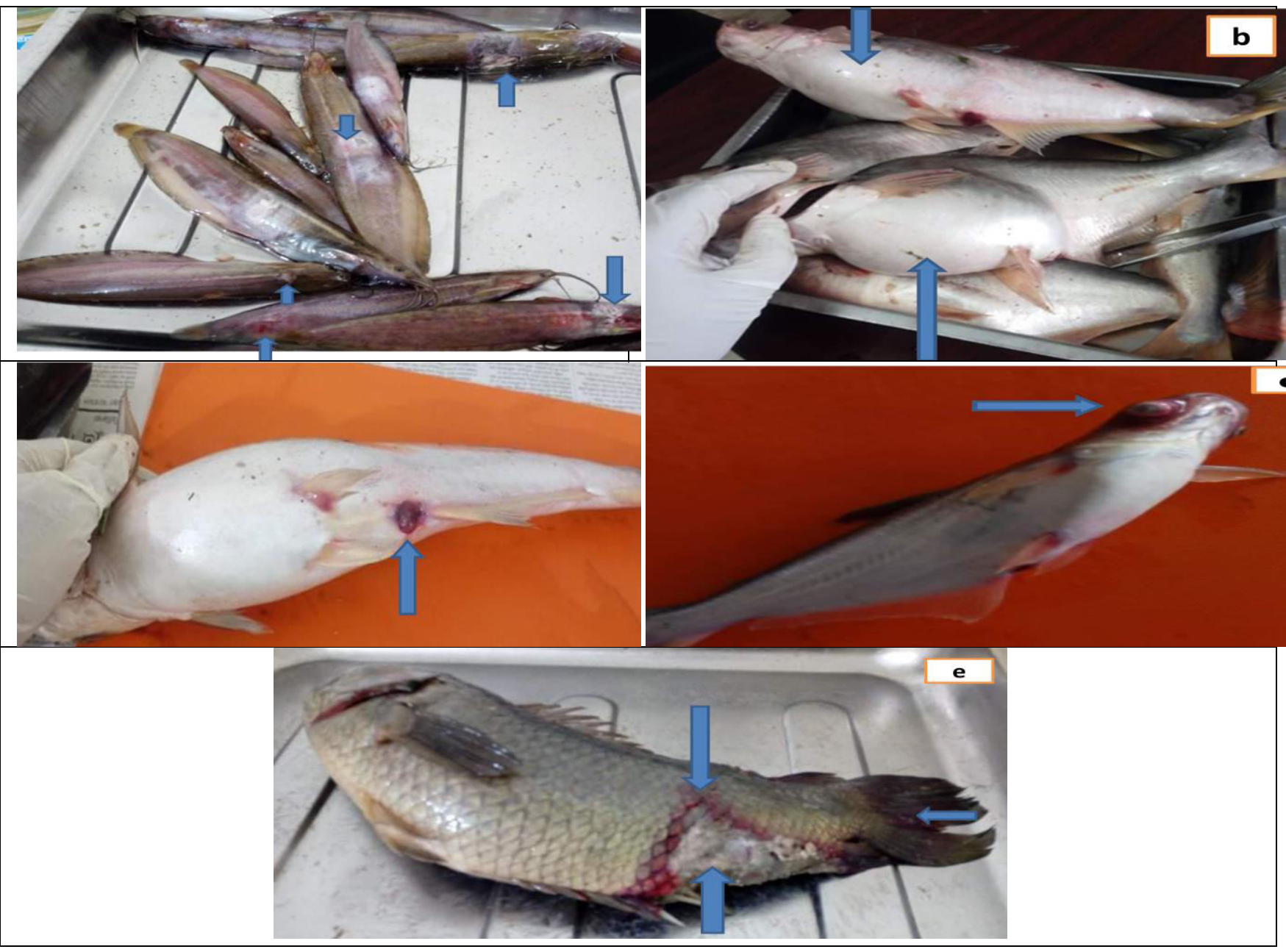

Fig 1: Infected fish samples from farmer's ponds. (a) Shing (H. fossilis) with hemorrhagic ulcerative lesion, (b) Pangas (P. pangasius) with dropsy, (c) Pangas (P. pangasius) with rectal protrusion, (d) Pabda (Ompok spp) with pop eye and reddish discoloration around the eye and mouth, (e) Koi (A.testudineus) with deep haemorrhagic ulcerative lesion on tail region.

Table 1: Bacteria isolated from different infected fishes.

\begin{tabular}{l|c|c|c|c|c|c}
\hline \multirow{2}{*}{ Fish pathogens } & \multicolumn{6}{|c}{ Name of fishes: N=95 (\%) } \\
\cline { 2 - 7 } & Shing & Pangas & Pabda & Koi & $\begin{array}{c}\text { Other } \\
\text { fishes }\end{array}$ & $\begin{array}{c}\text { Total isolated } \\
\text { bacterial pathogens }\end{array}$ \\
\hline Aeromonas spp & 23 & 2 & 1 & 4 & 6 & $36(42.9 \%)$ \\
\hline Pseudomonas spp & 13 & 0 & 0 & 1 & 1 & $15(17.9 \%)$ \\
\hline Vibrio spp. & 1 & 0 & 5 & 0 & 1 & $7(8.3 \%)$ \\
\hline Staphylococcus spp & 5 & 1 & 0 & 2 & 1 & $9(10.7 \%)$ \\
\hline Flavobacterium spp & 2 & 5 & 0 & 0 & 0 & $7(8.3 \%)$ \\
\hline Citobacter spp, & 2 & 0 & 0 & 0 & 0 & $2(2.4 \%)$ \\
\hline Edwardsella spp & 1 & 5 & 0 & 0 & 1 & $7(8.3 \%)$ \\
\hline Enterobacter spp & 0 & 0 & 0 & 0 & 1 & $1(1.2 \%)$ \\
\hline $\begin{array}{l}\text { Total isolated bacterial } \\
\text { pathogens fishes }(\%)\end{array}$ & $47(49.5 \%)$ & $13(13.7 \%)$ & $6(6.3 \%)$ & $7(7.3 \%)$ & $11(11.6 \%)$ & $\mathbf{8 4}(\mathbf{8 8 . 4 \% )}$ \\
\hline Normal flora & 7 & 1 & 3 & 0 & 0 & $\mathbf{1 1}(\mathbf{1 1 . 6 \% )}$ \\
\hline Total isolates & $54(56.9 \%)$ & $14(14.7 \%)$ & $9(9.5 \%)$ & $7(7.3 \%)$ & $11(11.6 \%)$ & $95(100 \%)$ \\
\hline
\end{tabular}


Out of eight (8) isolates only four strains namely Aeromonas spp., Vibrio spp., Citobacter spp., Edwardsiella spp could ferment only glucose and peptone catabolized and showed alkaline (red color) and acidic (yellow) butt. Only Pseudomonas spp was unable to ferment glucose and expressed all stant and butt were red in color. There was only one strain of Gram positive cocci that was able to ferment glucose, lactose and sucrose and produced yellow color stant and butt. Flavobacterium spp exhibited little bit different pattern and that was acidic reaction in slant and weak positive butt. The isolated bacteria Aeromonas spp showed alkaline reaction in stant, acidic reaction in butt, gas production, motility, indole production, oxidase test, MR-VP, Simon citrate test, catalase test were positive whereas $\mathrm{H}_{2} \mathrm{~S}$ production, urea hydrolysis test were negative. Various strains of Pseudomonas spp showed motile in motility test, Simon citrate and catalase were positive. The carbohydrate utilization presented alkaline reaction in both in stant and butt and both were red in color. These strains also showed only MR-VP test, $\mathrm{H}_{2} \mathrm{~S}$ and gas production were negative. The strains of Vibrio spp showed positive motile, indole, oxidase, and Simon citrate test whereas their gas, $\mathrm{H}_{2} \mathrm{~S}$, urea production and MR test were negative. Out of the 7 isolated strains it was found that $\mathrm{H}_{2} \mathrm{~S}$ production by Citobacter spp, urea production by Pseudomonas spp and Citobacter spp, Simon citrate test by Aeromonas spp and VP tests by Aeromonas spp and Vibrio spp were variable respectively.

Table 2: Results of biochemical tests of the isolated bacterial species from infected fishes.

\begin{tabular}{|c|c|c|c|c|c|c|c|c|c|c|c|c|c|}
\hline \multirow[b]{2}{*}{ 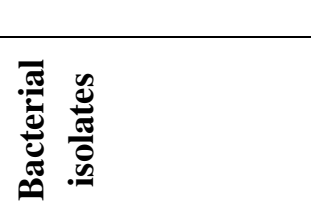 } & \multirow[b]{2}{*}{ ‡ } & \multicolumn{4}{|c|}{ KIA } & \multicolumn{3}{|c|}{ MIU medium } & \multirow[b]{2}{*}{ 造 } & \multirow[b]{2}{*}{ 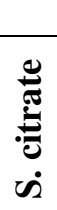 } & \multirow[b]{2}{*}{$\stackrel{\underline{z}}{\Sigma}$} & \multirow[b]{2}{*}{$\vec{b}$} & \multirow[b]{2}{*}{$\overrightarrow{\tilde{E}}$} \\
\hline & & 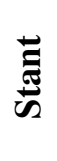 & $\stackrel{\Xi}{\Xi}$ & $\overbrace{\tilde{\sigma}}^{\mathscr{2}}$ & $\stackrel{\pi}{=}$ & $\overrightarrow{\mathrm{e}}$ & $\frac{\stackrel{0}{O}}{\frac{O}{g}}$ & 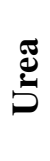 & & & & & \\
\hline Aeromonas spp & Gram negative & $\mathrm{R}$ & $\mathrm{Y}$ & + & - & + & + & - & + & \pm & + & \pm & + \\
\hline Pseudomonas spp & Gram negative & $\mathrm{R}$ & $\mathrm{R}$ & - & - & + & - & \pm & + & + & - & - & + \\
\hline Vibrio spp. & Gram negative & $\mathrm{R}$ & $\mathrm{Y}$ & - & - & + & + & - & + & + & - & \pm & + \\
\hline Staphylococcus spp & Gram positive & $\mathrm{Y}$ & $\mathrm{Y}$ & - & - & + & - & + & - & + & + & + & + \\
\hline Flavobacterium spp & Gram negative & Y & $\mathrm{W}$ & - & - & + & - & - & + & + & + & - & + \\
\hline Citobacter spp, & Gram negative & $\mathrm{R}$ & $\mathrm{Y}$ & + & \pm & + & + & \pm & - & + & + & - & + \\
\hline Edwardsiella spp. & Gram negative & $\mathrm{R}$ & $\mathrm{Y}$ & + & + & + & + & - & - & - & + & - & + \\
\hline
\end{tabular}

$(+)=$ Positive; $(-)=$ Negative reaction; $( \pm)=$ Variable; R=Red (Alkaline reaction);Y=Yellow (Acid reaction); W= Weak positive, $\mathrm{H}_{2} \mathrm{~S}=$ Hydrogen sulphide (Blackening); $\mathrm{MR}=$ Methyl Red, VP= Voges Proskaure, KIA=Kligler Iron agar, MIU=Motility indole urea test. Cat=Catalase test, Mot=Motility test,

\subsection{Susceptibility to antimicrobial agents' in-vitro} condition - In our experiment, all of the isolated pathogenic bacteria were $84 / 84(100 \%)$ resistant to Amoxicillin and 18/84 (64.3\%) resistant to Erythromycin. All the strains showed sensitive to Ciprofloxacin, Cotrimoxazole, Enorfloxacin, Doxyciline, Clotetracycline, and Colistin were 78/84 (92.8\%), 76/84 (90.5\%), 73/84(86.9\%), 67/84 (67.9\%), $53 / 84(63.1 \%)$ and 52/84(61.9\%) respectively. Colistin, Erythromycin and Clotetracycline expressed intermediate sensitivity were very few such as $33.3 \%, 26.2 \%$ and $15.5 \%$ respectively presented in Fig 2. Table 3-7 showed antibiotic sensitivity patterns all of the individual isolates.

\subsection{Physico-chemical characteristic of pond water -}

The recorded pond water parameters of 95 sub-surface water samples were studied shown in Table 8. The average range of temperature, $\mathrm{p}^{\mathrm{H}}$, ammonia, hardness, alkalinity, nitrate and nitrite varied depending on their cultivated fish types.

All of the fish water samples showed ammonia concentration higher than their normal value and that was $<0.5 \mathrm{mg} / \mathrm{L}$. Only Pangas fish pond water expressed the average hardness and Nitrite $\left(\mathrm{NO}_{2}\right)$ were 126.42 and $0.37 \mathrm{mg} / \mathrm{L}$ respectively that was higher than ideal value $20-100 \mathrm{mg} / \mathrm{L}$ and $<0.2 \mathrm{mg} / \mathrm{L}$ respectively Table 8 . 


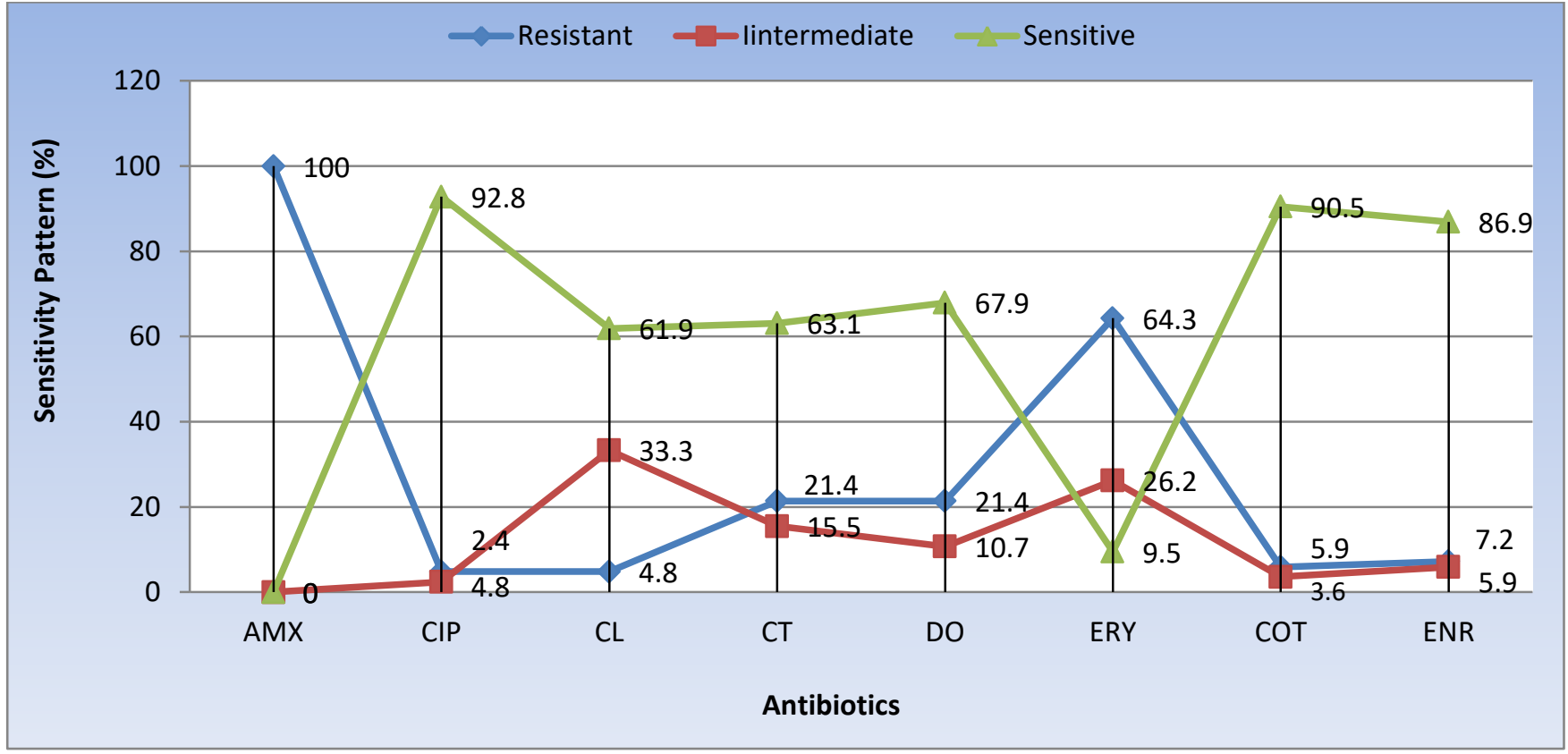

Fig 2: Antibiotic sensitivity pattern of all isolates. $A M X=A$ moxycillin, $C I P=C i p r o f l o x a c i n, ~ C L=C o l i s t i n$, $\mathrm{CT}=$ Clotetracycline, $\mathrm{DO}=$ Doxycycline, Ery= Erythromycine, $\mathrm{COT}=$ Cotrimoxazole, ENR= Enorfloxacin.

Table 3: In-vitro Antibiogram profile of Gram negative and Gram positive bacterial isolates from Shing fishes.

\begin{tabular}{|c|c|c|c|c|c|c|c|c|c|}
\hline \multirow{2}{*}{ Isolated bacteria } & & \multicolumn{8}{|c|}{ No $(\%)$} \\
\hline & & AMX & CIP & $\mathbf{C L}$ & CT & DO & ERY & COT & ENR \\
\hline \multirow{3}{*}{$\begin{array}{l}\text { Aeromonas } \\
\text { hydraphila } \\
(\mathrm{n}=23)\end{array}$} & $\mathrm{R}$ & $23(100)$ & $0(0)$ & $4(17.4)$ & $7(30.4)$ & $3(13.0)$ & $21(91.3)$ & $22(95.7)$ & $1(4.3)$ \\
\hline & $\mathrm{S}$ & $0(0)$ & $22(95.7)$ & $9(39.1)$ & $2(8.7)$ & $6(26.0)$ & $2(8.7)$ & $1(4.3)$ & $2(8.7)$ \\
\hline & I & $0(0)$ & $1(4.3)$ & $10(43.5)$ & $14(60.9)$ & 14 (60.9) & $0(0)$ & $0(0)$ & $20(87)$ \\
\hline \multirow{3}{*}{$\begin{array}{l}\text { Pseudomonas } \\
(\mathrm{n}=13)\end{array}$} & $\mathrm{R}$ & $13(100)$ & $0(0)$ & $0(0)$ & $2(15.4)$ & $1(7.7)$ & $12(92.3)$ & $1(7.7)$ & $0(0)$ \\
\hline & $\mathrm{S}$ & $0(0)$ & $13(100)$ & $9(69.2)$ & $8(61.5)$ & $9(69.2)$ & $1(7.7)$ & $12(92.3)$ & $13(100)$ \\
\hline & I & $0(0)$ & $0(0)$ & $4(30.8)$ & $3(23.1)$ & $3(23.1)$ & $0(0)$ & $0(0)$ & $0(0)$ \\
\hline \multirow{3}{*}{$\begin{array}{l}\text { Staphylococcus } \\
(\mathrm{n}=5)\end{array}$} & $\mathrm{R}$ & $5(100)$ & $0(0)$ & $1(20)$ & $1(20)$ & $2(40)$ & $5(100)$ & $0(0)$ & $0(0)$ \\
\hline & $\mathrm{S}$ & $0(0)$ & $5(100)$ & $2(40)$ & $3(60)$ & $0(0)$ & $0(0)$ & $5(100)$ & $4(80)$ \\
\hline & I & $0(0)$ & $0(0)$ & $2(40)$ & $1(20)$ & $3(60)$ & $0(0)$ & $0(0)$ & $1(20)$ \\
\hline \multirow{3}{*}{$\begin{array}{l}\text { Flavobacterium } \\
(\mathrm{n}=2)\end{array}$} & $\mathrm{R}$ & $2(100)$ & $0(0)$ & $0(0)$ & $1(50)$ & $0(0)$ & $1(50)$ & $1(50)$ & $0(0)$ \\
\hline & $\mathrm{S}$ & $0(0)$ & $2(100)$ & $2(100)$ & 1(50) & $1(50)$ & $0(0)$ & 1(50) & $1(50)$ \\
\hline & I & $0(0)$ & $00(0)$ & $0(0)$ & $0(0)$ & $1(50)$ & $1(50)$ & $0(0)$ & $1(50)$ \\
\hline \multirow{3}{*}{$\begin{array}{l}\text { Citrobacter spp. } \\
(\mathrm{n}=2)\end{array}$} & $\mathrm{R}$ & $2(100)$ & $1(50)$ & $0(0)$ & $0(0)$ & $0(0)$ & $2(100)$ & $0(0)$ & $1(50)$ \\
\hline & $\mathrm{S}$ & $0(0)$ & $1(50)$ & $2(100)$ & $2(100)$ & $2(100)$ & $0(0)$ & $2(100)$ & $1(50)$ \\
\hline & I & $0(0)$ & $0(0)$ & $0(0)$ & $0(0)$ & $0(0)$ & $0(0)$ & $0(0)$ & $0(0)$ \\
\hline \multirow{3}{*}{$\begin{array}{l}\text { Others }(\mathrm{n}=2, \\
\text { Vibrio spp. \& } \\
\text { Edwardsella spp) }\end{array}$} & $\mathrm{R}$ & $2(100)$ & $0(0)$ & $0(0)$ & $0(0)$ & $0(0)$ & $1(50)$ & $1(50)$ & $0(0)$ \\
\hline & $\mathrm{S}$ & $0(0)$ & $2(100)$ & $1(50)$ & $2(100)$ & $2(100)$ & $0(0)$ & $1(50)$ & $2(100)$ \\
\hline & I & $0(0)$ & $0(0)$ & $1(50)$ & $0(0)$ & $0(0)$ & 1(50) & $0(0)$ & $0(0)$ \\
\hline
\end{tabular}

AMX=Amoxycillin, $\quad \mathrm{CIP}=$ Ciprofloxacin, $\mathrm{CL}=$ Colistin, $\mathrm{CT}=$ Clotetracyclin, $\mathrm{DO}=$ Doxycillin, ERT=Erythomycin, COT=Trimethoprime, ENR=Enrofloxacin, $\mathrm{S}=$ Sensitive, $\mathrm{R}=$ Resistant, I=Intermediate 
Abedin et al., / American Journal of Pure and Applied Biosciences, 2(3), 39-50, 2020

Table 4: In-vitro Antibiogram profile of Gram negative and Gram positive bacterial isolates from Pangas fishes.

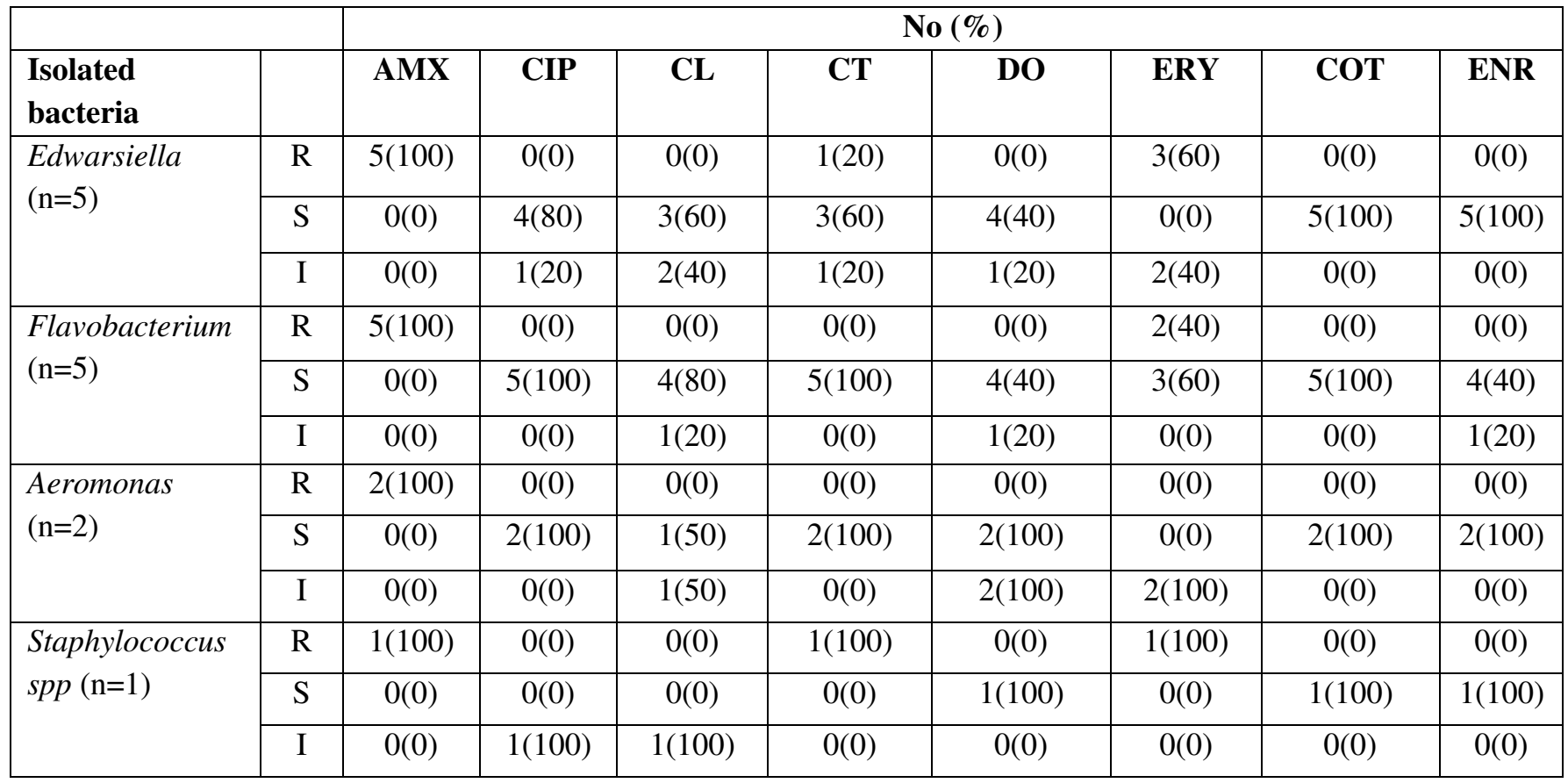

Table 5: In-vitro Antibiogram profile of Gram negative and Gram positive bacterial isolates from Koi fishes.

\begin{tabular}{|l|c|c|c|c|c|c|c|c|c|}
\hline \multicolumn{2}{|c|}{} & \multicolumn{9}{|c|}{ No (\%) } \\
\hline $\begin{array}{l}\text { Isolated } \\
\text { bacteria }\end{array}$ & & AMX & CIP & CL & CT & DO & ERY & COT & ENR \\
\hline $\begin{array}{l}\text { Aeromonas } \\
(\mathrm{n}=4)\end{array}$ & $\mathrm{R}$ & $4(100)$ & $1(25)$ & $1(25)$ & $0(0)$ & $2(50)$ & $0(0)$ & $0(0)$ & $2(50)$ \\
\cline { 2 - 11 } & $\mathrm{S}$ & $0(0)$ & $3(75)$ & $2(50)$ & $2(50)$ & $2(50)$ & $2(50)$ & $4(100)$ & $2(50)$ \\
\cline { 2 - 11 } & $\mathrm{I}$ & $0(0)$ & $0(0)$ & $1(25)$ & $2(50)$ & $0(0)$ & $2(50)$ & $0(0)$ & $0(0)$ \\
\hline $\begin{array}{l}\text { Staphylococcus } \\
\text { spp.(n=2) }\end{array}$ & $\mathrm{R}$ & $2(100)$ & $0(0)$ & $1(50)$ & $0(0)$ & $0(0)$ & $0(0)$ & $0(0)$ & $0(0)$ \\
\cline { 2 - 11 } & $\mathrm{S}$ & $0(0)$ & $2(100)$ & $0(0)$ & $1(50)$ & $2(100)$ & $0(0)$ & $1(50)$ & $2(100)$ \\
\cline { 2 - 10 } & $\mathrm{I}$ & $0(0)$ & $0(0)$ & $1(50)$ & $1(50)$ & $0(0)$ & $2(100)$ & $1(50)$ & $0(0)$ \\
\hline $\begin{array}{l}\text { Pseudomonas } \\
\text { (n=1) }\end{array}$ & $\mathrm{R}$ & $1(100)$ & $0(0)$ & $0(0)$ & 1() & $0(0)$ & $0(0)$ & $0(0)$ & $0(0)$ \\
\cline { 2 - 10 } & $\mathrm{S}$ & $0(0)$ & $1(100)$ & $0(0)$ & $0(0)$ & $0(0)$ & $0(0)$ & $1(100)$ & $1(100)$ \\
\cline { 2 - 10 } & $\mathrm{I}$ & $0(0)$ & $0(0)$ & $1(100)$ & $0(0)$ & $1(100)$ & $1(100)$ & $0(0)$ & $0(0)$ \\
\hline
\end{tabular}

Table 6: In-vitro Antibiogram profile of Gram negative and Gram positive bacterial isolates from Pabda fishes.

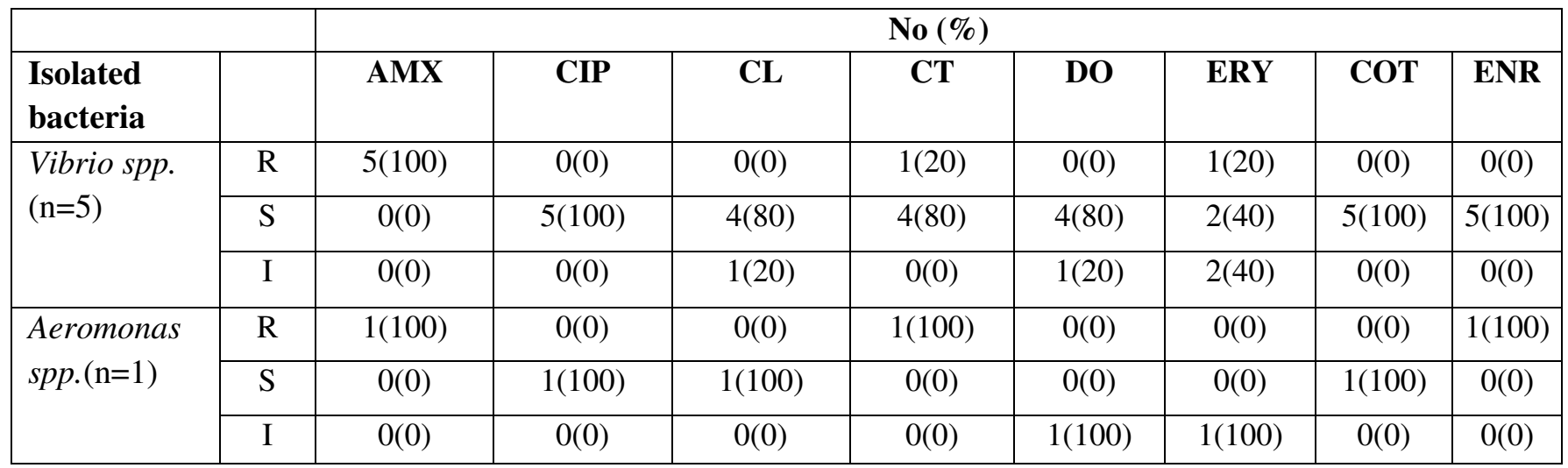


Abedin et al., / American Journal of Pure and Applied Biosciences, 2(3), 39-50, 2020

Table 7: In-vitro Antibiogram profile of Gram negative and Gram positive bacterial isolates from other fishes.

\begin{tabular}{|c|c|c|c|c|c|c|c|c|c|}
\hline & & \multicolumn{8}{|c|}{ No (\%) } \\
\hline $\begin{array}{l}\text { Isolated } \\
\text { bacteria }\end{array}$ & & AMX & CIP & CL & CT & DO & ERY & COT & ENR \\
\hline \multirow{3}{*}{$\begin{array}{l}\text { Aeromonas } \\
\text { spp. }(\mathrm{n}=6)\end{array}$} & $\mathrm{R}$ & $6(100)$ & $1(16.7)$ & $0(0)$ & $2(33.3)$ & $1(16.7)$ & $6(100)$ & $1(16.7)$ & $0(0)$ \\
\hline & $\mathrm{S}$ & $0(0)$ & $5(83.3)$ & $4(66.7)$ & $3(50)$ & $2(33.3)$ & $0(0)$ & $5(83.3)$ & $6(100)$ \\
\hline & I & $0(0)$ & $0(0)$ & $2(33.3)$ & $1(16.7)$ & $3(50)$ & $0(0)$ & $0(0)$ & $0(0)$ \\
\hline \multirow{3}{*}{$\begin{array}{l}\text { Other isolates } \\
(\mathrm{n}=5)\end{array}$} & $\mathrm{R}$ & $5(100)$ & $0(0)$ & $0(0)$ & $0(0)$ & $1(20)$ & $3(60)$ & $1(20)$ & $1(20)$ \\
\hline & $\mathrm{S}$ & $0(0)$ & $5(100)$ & $5(100)$ & $4(80)$ & $4(80)$ & $0(0)$ & $4(80)$ & $4(80)$ \\
\hline & I & $0(0)$ & $0(0)$ & $0(0)$ & $1(20)$ & $0(0)$ & $2(40)$ & $0(0)$ & $0(0)$ \\
\hline
\end{tabular}

Table 8: Physico-chemical parameters of water samples collected at a time during April to December 2019.

\begin{tabular}{|c|c|c|c|c|c|c|c|}
\hline 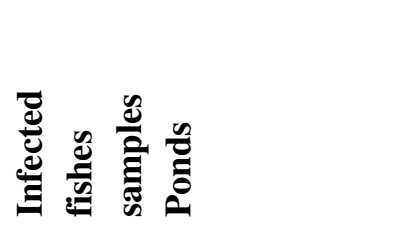 & 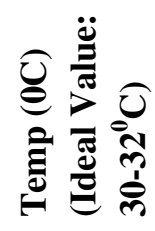 & 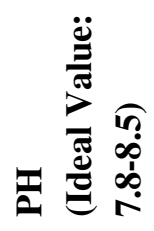 & 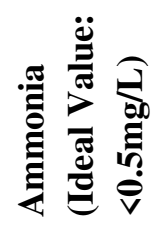 & 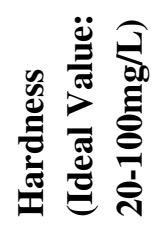 & 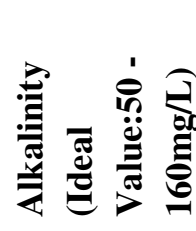 & 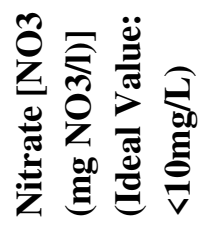 & 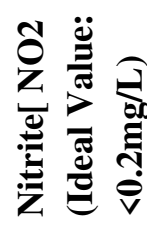 \\
\hline Shing fishes ponds water & 27.3 & 7.6 & 0.87 & 73.68 & 94.4 & 7.24 & 0.18 \\
\hline Pangas fishes ponds water & 26 & 7.2 & 0.9 & 126.42 & 139.85 & 10.7 & 0.37 \\
\hline Pabda fishes ponds water & 27.3 & 7.8 & 0.88 & 88.00 & 127.11 & 6.88 & 0.22 \\
\hline $\begin{array}{l}\text { Thai Koi fishes ponds } \\
\text { water }\end{array}$ & 27.0 & 7.7 & 1.87 & 76.28 & 140.14 & 7.64 & 0.18 \\
\hline Others fishes ponds water & 26.0 & 7.5 & 1.17 & 99.14 & 126.42 & 9.14 & 0.21 \\
\hline
\end{tabular}

\section{DISCUSSIONS}

Now not only in Bangladesh, but all over the world our local fishes have great demands in the global market due to their high nutritional quality. The cultivation of these fishes in pond water has been badly influenced by different factors including various bacterial, viral and fungal contagious pathogens (Sharif et al., 2019). The mortality rate in adult Shing, Pangas, Thai Koi, Pabda and others local fishes like Shol, Magur, Tilapia and Tengara were noticed in freshwater cultured ponds situated in Bangladesh. A few numbers of bacterial species are pathogenic to fishes, which incorporated Aeromonas spp, Pseudomonas spp, Vibrio spp, Staphylococcus spp, Flavobacterium spp, Edwardsiella spp, Citobacter spp, and Enterobacter spp (Shayoet et. al., 2012; Anshary et al., 2014).

However, a total of $84(88.4 \%)$ pathogenic bacteria and $11(11.6 \%)$ non- pathogenic bacteria were isolated, among pathogenic bacteria Gram positive cocci and Gram negative bacilli were 9(10.7\%) and 75 (89.7\%) respectively. A wide range of bacterial species has been recovered from local fishes in our work including Aeromonas spp, Pseudomonas spp, Vibrio spp, Staphylococcus spp, Flavobacterium spp, Edwardsiella spp, with frequency of 36 (42.9\%), 15(17.9\%), 7(8.3\%) 9(10.7\%) 7(8.3\%) and 7(8.3\%) respectively and others were $3(3.6 \%)$ Citobacter spp and Enterobacter spp, similar findings have also been reported from other districts of Bangladesh (Al-Harbi and Uddin, 2005; Amal et al., 2010; and Marcel et al., 2013).

Aeromonas spp, Pseudomonas strains and other isolates were identified from aquaculture pond fishes reported by Hossian and Chowdhury (2009). In Bangladesh, detached and distinguished some Pseudomonas strains from unhealthy cultivated fishes of Bangladesh. The different types of bacterial species of the pond water fishes (Shing) were isolated by numerous scientists (Shewan, 2000; and Okaeme, 2006). Ahmed and Shoreit (2001) also reported that 
Abedin et al., / American Journal of Pure and Applied Biosciences, 2(3), 39-50, 2020

mixed bacterial contaminations occurred by Aeromonas spp and Pseudomonas spp. Shing ( $H$. fossilis) is most demandable and highly valuable fish in Bangladesh. Although, the mortality rate of Shing is very low but its cultivation in pond water is influenced by different factors such as bacterial pathogens (Shahen et al., 2019). In any case, the clinical manifestations were loss of balance, hemorrhages, skin sores, body and tail disintegration, mucous discharge, and blockage and development with drain of the inside organs which were comparable with the discoveries of Alicia et al. 2005; Khalil et al. 2010; and Mastan, 2013. However, the predominant pathogenic bacteria isolated from the pond cultured Shing were Gramnegative bacteria (50.0\%), as correspondingly observed by Marcel et al., 2013. Moreover, in Shing fish, the isolated pathogenic bacteria were Aeromonas spp 23(48.9\%), Pseudomonas spp 13(27.7\%), Gram positive cocci Staphylococcus spp 5(10.6\%) and other pathogens including Vibrio spp, Flavobacteriem spp, Citobacter spp and Edwardsella spp were 6(12.8\%). Aeromonas spp were the highly isolated bacteria in this study. Among the pathogenic bacterial diseases, Aeromonas spp were the major pathogens that induced plagues of ulcerative ailment in fish in Southeast Asia and different areas of the world (Anyanwu et al., 2014).

Bacterial isolates of Pseudomonas spp from Shing $(H$. fossilis) were $100 \%$ susceptible to Enorfloxacin and Ciprofloxacin, Staphylococcus spp to Ciprofloxacin and Cotrimoxazole, Flavobacterium spp to Ciprofloxacin and Colistin, Citrobacter spp to Colistin, Clotetracyclin, Doxycycline, Cotrimoxazole and Vibrio spp and Edwardsella spp to Ciprofloxacin, Clotetracyclin, Doxycycline, and Enorfloxacin. Highly sensitive to Ciprofloxacin (95.7\%) and Cotrimoxazole (92.3\%) were observed in Aeromonas spp and Pseudomonas spp respectively and poor sensitive to Colistin (69.2\%), Clotetracyclin (61.5\%), Doxycycline (69.2\%) in Pseudomonas spp., and Clotetracyclin (60\%) and Enorfloxacin (80\%) in Staphylococcus spp were found. In our study, the highly intermediate sensitive were to Clotetracyclin (60.9\%), Doxycyline (60.9\%), and Enrofloxacin (87.0\%) in Aeromonas spp; Doxycyline $(60.0 \%)$ in Staphylococcus spp was observed. In our investigation was observed all bacterial isolates were highly resistance to amoxicillin
(100\%). Moreover, Staphylococcus spp and Citobacter spp were $100 \%$ resistant to Erythromycin in our study. The resistant pattern of Erythromycin (91.3\%) and Cotrimoxazole (95.7\%) were found in Aeromonas spp; Erythromycin (92.3\%) in Pseudomonas spp.

Now a day, in Bangladesh, most available cultivated fishes are panagas ( $P$. pangasius). The isolated bacteria were Flavobacterium spp 5(38.5\%), Edwardsella spp 5(38.5\%), Aeromonas spp 2(15.4\%). The $100 \%$ sensitive bacterial isolates were Edwarsiella spp to Cotrimoxazole and Enorfloxacin; Flavobacterium spp to Clotetracyclin, Ciprofloxacin, and Cotrimoxazole; Aeromonas spp to Clotetracyclin, Ciprofloxacin, Doxycycline, Cotrimoxazole and Enorfloxacin, and only one strain of Staphylococcus spp to Doxycycline, Cotrimoxazole and Enorfloxacin, where Edwarsiella spp. showed poor sensitive to Ciprofloxacin (80\%), Colistin (60\%), and Clotetracyclin (60\%), and only one strain of Flavobacterium spp showed $60 \%$ sensitivity to Enorfloxacin. In this investigation, only one strain of Edwarsiella spp was observed to $60 \%$ resistant to Erythromycin. The resistant pattern of Staphylococcus spp was $100 \%$ against Clotetracyclin and Erythromycin (Islam et al., 2020).

Thai Koi (A. testudineus) is the most available and cheapable fishes in Bangladesh. The pond water cultivated Thai Koi fishes were infected with some bacterial pathogens namely by Aeromonas spp were 4(57.1\%) and secondly by Staphylococcus spp were $2(28.6 \%)$ in this study. Comparable isolation rates were accounted by Shittu et al. (2009). In our investigation, Aeromonas spp expressed $100 \%$ sensitivity to Cotrimoxazole and $75 \%$ to Ciprofloxacin, whereas Staphylococcus spp were 100\% sensitive to Ciprofloxacin, Doxycycline and Enorfloxacin, Cotrimoxazole; third one Pseudomonas spp were $100 \%$ sensitive to Ciprofloxacin, Cotrimoxazole and Enorfloxacin. The parameters of intermediate sensitivity were $100 \%$ to Erythromycin in Staphylococcus spp, and Colistin, Doxycycline and Erythromycin were observed in Pseudomonas spp. The significant level of resistance from regularly utilized anti-microbials is similar with Hussain et al. (2014) and Mostafa et al. (2008). 
Vibrio spp $(83.3 \%)$ were the most predominant Gram negative bacteria and Aeromonas spp (16.3\%) were the second common isolated bacteria found in Pabda fish (Ompok spp) in our work. In this study, bacterial isolates Vibrio spp were $100 \%$ sensitive to Cotrimoxazole and Enorfloxacin; Aeromonas spp were $100 \%$ sensitive to Cotrimoxazole and Ciprofloxacin; Flavobacterium spp were $100 \%$ sensitive to Colistin, Ciprofloxacin, and Cotrimoxazole. Vibrio spp were highly sensitive $(80 \%)$ to Colistin, Clotetracyclin and Doxycycline, whereas highly intermediate sensitive were to only Erythromycin (100\%). Our findings are not correspondent with different examinations led by Simu et al. (2019).

In our examination of other fish spp, 6 (54.5\%) of Aeromonas spp were found in other spp of deshi fishes such as Shol (C. striata), Magur (Clarias spp), Tilapia (O. niloticus), and Tengara (M. cavasius) and the rest $5(45.5 \%)$ isolates were Pseudomonas spp, Vibrio spp, Staphylococcus spp, Edwardsella spp, and Enterobacter spp. In other fish spp such as Shol, Magur, Tilapia, and Tengara, bacterial isolates mainly Aeromonas spp were $100 \%$ sensitive to Enorfloxacin and low sensitive to Ciprofloxacin (83.3\%), Colistin (66.7\%), and Cotrimoxazole(83.3\%). Other isolates (Citobacter spp, Enterobacter spp) were $100 \%$ sensitive to Ciprofloxacin and Colistin, and low sensitive to $80 \%$ Clotetracyclin, Doxycycline, Cotrimoxazole and Enorfloxacin. All of the strains showed 100\% resistant to Amoxycillin. Similar results were recorded by Hussain et al. (2014) and Mostafa et al. (2008).

The water quality influenced by the encompassing of ecological conditions may affect the pond cultured fishes. This in the end diminishes the insusceptible status, activating bacterial contamination prompting infection episodes (Amal et al., 2015). The fish wellbeing status and pond cultured environments were reflected due to several bacterial flora. Similar study was conducted by Pakingking et al. (2015). The lake water's physiochemical parameters such as ammonia, hardness, alkalinity, nitrate, nitrite, temperature, and $\mathrm{pH}$ were significant in our study area (Ahmad et al., 2018). In this study, every inspecting site had distinctive characteristics that were related with the nearness of microorganisms. This phenomenon was also observed in multiple works done by other investigators. This investigation uncovered that alkali impacted the presence of different pathogenic bacterial spp at all of the sampling areas. It is believed that the ammonia in ponds water comes from fish excrement and exorbitant feed given to the fish.

\section{CONCLUSION}

This study has clarified that poor water quality and bacterial diseases could be a major cause of considerable economic loss to fish farmers in greater Mymensingh, Bangladesh. A number of bacterial spp including Aeromonas spp Pseudomonas spp, Vibrio spp, Staphylococcus spp, Flavobacterium spp, Edwardsiella spp, Citobacter spp, and Enterobacter spp were the major causes of bacterial diseases to different fish spp. Clinical examination of diseased fishes indicated that due to bacterial infection some severe damages were found in fish bodies such as equilibrium loss, hemorrhagic ulcerative lesion, rectal protrusion, dropsy, body and tail erosion, reddish discoloration around the eye and mouth, skin lesions on body surface and profuse mucous secretion. The presence of antibiotic resistant isolates imposes a serious concern about the drug of choice for treatment in fishes. A careful consideration should be given before deciding the antibiotic for treatment so as to prevent the emergence of antibiotic resistance and properly maintain the physiochemical parameters in ponds water to reduce the mortality of aquaculture fishes in ponds water. Moreover, disease prevention of various local fishes should be carried out by employing better culture practices and health management to ensure the highest yields and the optimum quality of the products.

\section{ACKNOWLEDGEMENTS}

We thank Md. Hashmot Ali; Aqua Laboratory Quality feeds Limited for his assistance in the laboratory analysis. We would like to special thank Md Rezanur Rahaman, Department of Biochemistry and Biotechnology at KYAU for his expert advice and encouragement throughout this difficult study.

\section{CONFLICTS OF INTEREST}

The authors announce that there is no conflict of interest with respect to the publication of this article. 


\section{REFERENCES}

1. Ahmad T, Uddin ME, Alam MK, Moniruzzaman M, Saha B, Sufian A, Alam M. G, Hossain I. Evaluation of Microbial and Physiochemical Properties of Three Selected Lakes Water in Dhaka City, Bangladesh. Scholars Academic J. of Biosciences, 6(2): 230-238, 2018. https://doi.org/10.21276/sajb.2018.6.2.17

2. Austin B. (2011). Taxonomy of bacterial fish pathogens. Vet. Res., 42(1): 1-13. https://doi.org/10.1186/1297-9716-42-20

3. Al-Harbi A.H., Uddin, N. (2005). Bacterial diversity of Tilapia (Oreochromis niloticus) cultured in brackish water in Saudi Arabia. Aquaculture, 250, 566-572. https://doi.org/10.1016/j.aquaculture.2005.01.026

4. Amal M.N.A., Zamri-Saad, M., Siti-Zahrah, A., Zulkafli, R., Misri, S., Nur-Nazifah, M.,Shahidan, H., (2010). Prevalence of Streptococcus agalactiae in Tilapia kept indifferent water bodies. Online J. Vet. Res. 11(2), 153-162.

5. Amal M.N.A., Zamri-Saad, M., Siti-Zahrah, S., Zulkafli, A.R. (2015). Water quality influences the presence of Streptococcus agalactiae in cage cultured red hybrid Tilapia, Oreochromis niloticus $\times$ Oreochromis mossambicus. Aquacult. Res. 46(2), 313-323.

https://doi.org/10.1111/are.12180

6. Anshary H., Kurniawan, R.A., Sriwulan, S., Ramli, R., Baxa, D.V., (2014). Isolation and molecular identification of the etiological agents of Streptococcosis in Niletilapia (Oreochromis niloticus) cultured in net cages in Lake Sentani, Papua, Indonesia, 3(1), 1-11. https://doi.org/10.1186/2193-1801-3-627

7. Alicia E, Toranzo A, Magarinos B, Jesus L, and Romalde R. (2005). A review of the main bacterial fish diseases in mariculture Systems. Aquaculture, 246: 37-46. https://doi.org/10.1016/j.aquaculture.2005.01.002

8. Anyanwu MU, Chah KF and Shoyinka VS. (2014). Antibiogram of aerobic bacteria isolated from skin lesions of African catfish cultured in Southeast Nigeria. International Journal of Fisheries and Aquatic Studies, 2: 134-141.

9. Ahmed SM and Shoreit AAM. (2001). Bacterial hemorrhagic septicemia in Oreochromis UniversePG I www.universepg.com niloticus at Aswan fish hatcheries. Assiut Vet. Med. J., 7(11): 950-961.

http://dx.doi.org/10.17582/journal.aavs/2019/7.11. 950.961

10. CLSI - Clinical and Laboratory Standards Institute, (2015). Performance standards for antimicrobial susceptibility testing. Twentysecond informational supplement. Wayne, PA, USA. CLSI.

11. Hossian MM and Chowdhury MR. (2009). Pseudomonas anguilliseptica as a pathogen of Tilapia (Oreochromis niloticus) culture in Bangladesh. Bangladesh Research Publication Journal, 4: 721-721.

12. Hussain MG. (2014). Global opportunity for fisheries and aquaculture in Bangladesh. Paper presented in the 2nd International Exhibition and Seminar Dairy, Aqua and Pet Animals-2014, Dhaka, Bangladesh.

13. Islam, R., Hossain, Md. N., Alam, Md. K., Uddin, Md. E., Rony, M. H., Imran, Md. A. S., and Alam, Md. F. (2020). Antibacterial activity of lactic acid bacteria and extraction of bacteriocin protein, Advances in Bioscience and Biotechnology, 11, 49-59. https://doi.org/10.4236/abb.2020.112004

14. John G, Holt-Noel R, Peter K, James S and Stanely T. (1998). Bergey's Manual of Determination Bacteriology. (Williams (Eds) $9^{\text {th }}$ Edn.). Lippincott Williams and Wilkins Publication, Philadelphia, PA.19106. USA.

15. Khalil SA, Khalil RH, Saad TT and Safaa MM. (2010). Studies on Pseudomonas septicemia among cultured Oreochromus niloticus. J. Aquacul. Assoc. Saudi Arabia, 5: 55-60.

16. Marcel G., Sabri, M.Y., Siti-Zahrah, A., Emikpe, B.O. (2013). Water condition and identification of potential pathogenic bacteria from red Tilapia reared in cage cultured system in two different water bodies in Malaysia. Afr. J. Microbiol. Res. 7(47), 5330-5337. https://doi.org/10.5897/AJMR12.1468

17. Mastan SA. (2013). Pseudomonas septicemia in Labeo rohita (ham.) and Cyprinus carpio (linn.) in and hra Pradesh natural occurrence and artificial challenge. International J. of Pharmacy and Pharmaceutical Sciences, 5: 564-568. 
18. Md. Ekhlas Uddin, Pulak Maitra, Hossain Md. Faruquee, Md. Firoz Alam, (2014). Isolation and characterization of proteases enzyme from locally isolated Bacillus sp., American Journal of Life Sciences. 2(6), 338-344. https://doi.org/10.11648/j.ajls.20140206.12

19. Mostafa M and Ahamed F. (2008). Pethogenesis of Aeromonas hydrophila on Heteropneustes fossilis. Bangladesh J. of Fis., 8: 38-41.

20. Okaeme AN. (2006). Fish diseases prevention and control paper presented at the $\mathrm{VCN}$ professional country education seminar Akure. 8, 1- 17.

21. Pakingking R.J., Palma, P., Usero, R. (2015). Quantitative and qualitative analyses of the bacterial microbiota of Tilapia (Oreochromis niloticus) cultured in earthen ponds in the Philippines. World J. Microbiol. Biotechnol. 31(2), 265-275. https://doi.org/10.1007/s11274-014-1758-1

22. Rahman MA, Ahmad T, Mahmud S, Barman NC, Haque MS, Uddin ME, and Ahmed R. (2019). Isolation, identification and antibiotic sensitivity pattern of Salmonella spp. from locally isolated egg samples, Am. J. Pure Appl. Sci., 1(1), 1-11. https://doi.org/10.34104/ajpab.019.019111

23. Shahen MZ, Mahmud S, Rony MH, Al Maruf MA, Uddin ME and Alam MS. (2019). Effect of antibiotic susceptibility and inhibitory activity for the control of growth and survival of microorganisms of extracts of Calendula officinalis, Eur. J. Med. Health Sci. 1(1), 1-9. https://doi.org/10.34104/ejmhs.019
24. Sharif IH, Haque MA, Jamal MAHM, and Uddin ME. (2019). Assessment and biomonitoring of the effect of rapeseeds oil on wister rat organs. Am. J. Pure Appl. Sci., 1(4), 20-29. https://doi.org/10.34104/ajpab.019.0192029

25. Shayoet S.D., Mwita, C.J., Hosea, K.M. (2012). Virulence of Pseudomonas and Aeromonas bacteria recovered from Oreochromis niloticus (Perege) from Mterahydropower Dam; Tanzania. Ann. Biol. Res. 3(11), 5157-5161. http://scholarsresearchlibrary.com/AB

26. Shewan JM. (2000). The Microbiology of sea water fish vol.1. Academic press, New York. pp. $487-560$.

27. Simu ZA et al. (2019). Investigation of a bacterial pathogen isolated from farmed Ompok pabda. Bangladesh J. Fish., 31(2): 243-252.

28. Shittu AO, Nubel U, Udo EE, Lin $J$ and Gaogakwe S. (2009). Characterization of methicillin-resistant Staphylococcus aureus (MRSA) isolates from hospitals in KwaZuluNatal (KZN) province, Republic of South Africa. J. Med. Microbiol., 58: 1219-1226. https://doi.org/10.1099/jmm.0.011452-0

29. Uddin M. E., Ahmad T., Moniruzzaman M., Ray S. K., and Ahammed T. (2017). Thermotolerant extracellular proteases produced by Bacillus subtilis isolated from local soil that representing industrial applications. J. of Pure and Applied Microbiol. 11(2), 733-741. https://doi.org/10.22207/JPAM.11.2.12

Citation: Abedin MZ, Rahman MS, Hasan R, Shathi JH, Jarin L, and Sifat Uz Zaman M. (2020). Isolation, identification, and antimicrobial profiling of bacteria from aquaculture fishes in pond water of Bangladesh. Am. J. Pure Appl. Sci., 2(3), 39-50. https://doi.org/10.34104/ajpab.020.039050 C) 